\title{
Neutral Operator and Neutral Differential Equation
}

\author{
Jingli Ren, ${ }^{1}$ Zhibo Cheng, $^{1}$ and Stefan Siegmund ${ }^{2}$ \\ ${ }^{1}$ Department of Mathematics, Zhengzhou University, Zhengzhou 450001, China \\ ${ }^{2}$ Department of Mathematics, Dresden University of Technology, 01062 Dresden, Germany
}

Correspondence should be addressed to Jingli Ren, renjl@zzu.edu.cn

Received 22 March 2011; Accepted 15 June 2011

Academic Editor: Allan C. Peterson

Copyright (C) 2011 Jingli Ren et al. This is an open access article distributed under the Creative Commons Attribution License, which permits unrestricted use, distribution, and reproduction in any medium, provided the original work is properly cited.

In this paper, we discuss the properties of the neutral operator $(A x)(t)=x(t)-c x(t-\delta(t))$, and by applying coincidence degree theory and fixed point index theory, we obtain sufficient conditions for the existence, multiplicity, and nonexistence of (positive) periodic solutions to two kinds of second-order differential equations with the prescribed neutral operator.

\section{Introduction}

In [1], Zhang discussed the properties of the neutral operator $\left(A_{1} x\right)(t)=x(\mathrm{t})-c x(t-\delta)$, which became an effective tool for the research on differential equations with this prescribed neutral operator, see, for example, [2-5]. Lu and Ge [6] investigated an extension of $A_{1}$, namely, the neutral operator $A_{2} x(t)=x(t)-\sum_{i=1}^{n} c_{i} x\left(t-\delta_{i}\right)$ and obtained the existence of periodic solutions for a corresponding neutral differential equation.

In this paper, we consider the neutral operator $(A x)(t)=x(t)-c x(t-\delta(t))$, where $c$ is constant and $|c| \neq 1, \delta \in C^{1}(\mathbb{R}, \mathbb{R})$, and $\delta$ is an $\omega$-periodic function for some $\omega>0$. Although $A$ is a natural generalization of the operator $A_{1}$, the class of neutral differential equation with $A$ typically possesses a more complicated nonlinearity than neutral differential equation with $A_{1}$ or $A_{2}$. For example, the neutral operators $A_{1}$ and $A_{2}$ are homogeneous in the following sense $\left(A_{i} x\right)^{\prime}(t)=\left(A_{i} x^{\prime}\right)(t)$ for $i=1,2$, whereas the neutral operator $A$ in general is inhomogeneous. As a consequence many of the new results for differential equations with the neutral operator $A$ will not be a direct extension of known theorems for neutral differential equations.

The paper is organized as follows: in Section 2, we first analyze qualitative properties of the neutral operator $A$ which will be helpful for further studies of differential equations 
with this neutral operator; in Section 3, by Mawhin's continuation theorem, we obtain the existence of periodic solutions for a second-order Rayleigh-type neutral differential equation; in Section 4, by an application of the fixed point index theorem we obtain sufficient conditions for the existence, multiplicity, and nonexistence of positive periodic solutions to second-order neutral differential equation. Several examples are also given to illustrate our results. Our results improve and extend the results in $[1,2,4,7]$.

\section{Analysis of the Generalized Neutral Operator}

Let $C_{\omega}=\{x \in C(\mathbb{R}, \mathbb{R}): x(t+\omega)=x(t), t \in \mathbb{R}\}$ with norm $\|x\|=\max _{t \in[0, \omega]}|x(t)|$. Then $\left(C_{\omega},\|\cdot\|\right)$ is a Banach space. A cone $K$ in $C_{\omega}$ is defined by $K=\left\{x \in C_{\omega}: x(t) \geq \alpha\|x\|\right.$, for all $\left.t \in \mathbb{R}\right\}$, where $\alpha$ is a fixed positive number with $\alpha<1$. Moreover, define operators $A, B: C_{\omega} \rightarrow C_{\omega}$ by

$$
(A x)(t)=x(t)-c x(t-\delta(t)), \quad(B x)(t)=c x(t-\delta(t)) .
$$

Lemma 2.1. If $|c| \neq 1$, then the operator $A$ has a continuous inverse $A^{-1}$ on $C_{\omega}$, satisfying

$$
\left(A^{-1} f\right)(t)=\left\{\begin{array}{l}
f(t)+\sum_{j=1}^{\infty} c^{j} f\left(s-\sum_{i=1}^{j-1} \delta\left(D_{i}\right)\right), \quad \text { for }|c|<1, \forall f \in C_{\omega} \\
-\frac{f(t+\delta(t))}{c}-\sum_{j=1}^{\infty}\left(1 / c^{j+1}\right) f\left(s+\delta(t)+\sum_{i=1}^{j-1} \delta\left(D_{i}\right)\right), \quad \text { for }|c|>1, \forall f \in C_{\omega} .
\end{array}\right.
$$

(2) $\left|\left(A^{-1} f\right)(t)\right| \leq\|f\| /|1-| c \|$, for all $f \in C_{\omega}$.

(3) $\int_{0}^{\omega}\left|\left(A^{-1} f\right)(t)\right| d t \leq 1 /|1-| c|| \int_{0}^{\omega}|f(t)| d t$, for all $f \in C_{\omega}$.

Proof. We have the following cases

Case $1(|c|<1)$. Let $t-\delta(t)=s$ and $D_{j}=s-\sum_{i=1}^{j-1} \delta\left(D_{i}\right), j=1,2, \ldots$. Therefore,

$$
\begin{gathered}
B^{j} x(t)=c^{j} x\left(s-\sum_{i=1}^{j-1} \delta\left(D_{i}\right)\right) \\
\sum_{j=0}^{\infty}\left(B^{j} f\right)(t)=f(t)+\sum_{j=1}^{\infty} c^{j} f\left(s-\sum_{i=1}^{j-1} \delta\left(D_{i}\right)\right) .
\end{gathered}
$$


Since $A=I-B$, we get from $\|B\| \leq|c|<1$ that $A$ has a continuous inverse $A^{-1}: C_{\omega} \rightarrow C_{\omega}$ with

$$
A^{-1}=(I-B)^{-1}=I+\sum_{j=1}^{\infty} B^{j}=\sum_{j=0}^{\infty} B^{j}
$$

where $B^{0}=I$. Then

$$
\left(A^{-1} f(t)\right)=\sum_{j=0}^{\infty}\left[B^{j} f\right](t)=\sum_{j=0}^{\infty} c^{j} f\left(s-\sum_{i=1}^{j-1} \delta\left(D_{i}\right)\right)
$$

and consequently

$$
\left|\left(A^{-1} f\right)(t)\right|=\left|\sum_{j=0}^{\infty}\left[B^{j} f\right](t)\right|=\left|\sum_{j=0}^{\infty} c^{j} f\left(s-\sum_{i=1}^{j-1} \delta\left(D_{i}\right)\right)\right| \leq \frac{\|f\|}{1-|c|} .
$$

Moreover,

$$
\begin{aligned}
\int_{0}^{\omega}\left|\left(A^{-1} f\right)(t)\right| d t & =\int_{0}^{\omega}\left|\sum_{j=0}^{\infty}\left(B^{j} f\right)(t)\right| d t \\
& \leq \sum_{j=0}^{\infty} \int_{0}^{\omega}\left|\left(B^{j} f\right)(t)\right| d t \\
& =\sum_{j=0}^{\infty} \int_{0}^{\omega}\left|c^{j} f\left(s-\sum_{i=1}^{j-1} \delta\left(D_{i}\right)\right)\right| d t \\
& \leq \frac{1}{1-|c|} \int_{0}^{\omega}|f(t)| d t .
\end{aligned}
$$

Case $2(|c|>1)$. Let

$$
\begin{gathered}
E: C_{\omega} \longrightarrow C_{\omega}, \quad(E x)(t)=x(t)-\frac{1}{c} x(t+\delta(t)), \\
B_{1}: C_{\omega} \longrightarrow C_{\omega}, \quad\left(B_{1} x\right)(t)=\frac{1}{c} x(t+\delta(t)) .
\end{gathered}
$$

By definition of the linear operator $B_{1}$, we have

$$
\left(B_{1}^{j} f\right)(t)=\frac{1}{c^{j}} f\left(s+\sum_{i=1}^{j-1} \delta\left(D_{i}\right)\right)
$$


where $D_{i}$ is defined as in Case 1 . Summing over $j$ yields

$$
\sum_{j=0}^{\infty}\left(B_{1}^{j} f\right)(t)=f(t)+\sum_{j=1}^{\infty} \frac{1}{c^{j}} f\left(s+\sum_{i=1}^{j-1} \delta\left(D_{i}\right)\right) .
$$

Since $\left\|B_{1}\right\|<1$, we obtain that the operator $E$ has a bounded inverse $E^{-1}$,

$$
E^{-1}: C_{\omega} \longrightarrow C_{\omega}, \quad E^{-1}=\left(I-B_{1}\right)^{-1}=I+\sum_{j=1} B_{1}^{j},
$$

and for all $f \in C_{\omega}$ we get

$$
\left(E^{-1} f\right)(t)=f(t)+\sum_{j=1}^{\infty}\left(B_{1}^{j} f\right)(t)
$$

On the other hand, from $(A x)(t)=x(t)-c x(t-\delta(t))$, we have

$$
(A x)(t)=x(t)-c x(t-\delta(t))=-c\left[x(t-\delta(t))-\frac{1}{c} x(t)\right]
$$

that is,

$$
(A x)(t)=-c(E x)(t-\delta(t)) .
$$

Let $f \in C_{\omega}$ be arbitrary. We are looking for $x$ such that

$$
(A x)(t)=f(t) .
$$

that is,

$$
-c(E x)(t-\delta(t))=f(t)
$$

Therefore,

$$
(E x)(t)=-\frac{f(t+\delta(t))}{c}=: f_{1}(t)
$$

and hence

$$
x(t)=\left(E^{-1} f_{1}\right)(t)=f_{1}(t)+\sum_{j=1}^{\infty}\left(B_{1}^{j} f_{1}\right)(t)=-\frac{f(t+\delta(t))}{c}-\sum_{j=1}^{\infty} B_{1}^{j} \frac{f(t+\delta(t))}{c},
$$


proving that $A^{-1}$ exists and satisfies

$$
\begin{gathered}
{\left[A^{-1} f\right](t)=-\frac{f(t+\delta(t))}{c}-\sum_{j=1}^{\infty} B_{1}^{j} \frac{f(t+\delta(t))}{c}=-\frac{f(t+\delta(t))}{c}-\sum_{j=1}^{\infty} \frac{1}{c^{j+1}} f\left(s+\delta(t)+\sum_{i=1}^{j-1} \delta\left(D_{i}\right)\right),} \\
\left|\left[A^{-1} f\right](t)\right|=\left|-\frac{f(t+\delta(t))}{c}-\sum_{j=1}^{\infty} \frac{1}{c^{j+1}} f\left(s+\delta(t)+\sum_{i=1}^{j-1} \delta\left(D_{i}\right)\right)\right| \leq \frac{\|f\|}{|c|-1} .
\end{gathered}
$$

Statements (1) and (2) are proved. From the above proof, (3) can easily be deduced.

Lemma 2.2. If $c<0$ and $|c|<\alpha$, one has for $y \in K$ that

$$
\frac{\alpha-|c|}{1-c^{2}}\|y\| \leq\left(A^{-1} y\right)(t) \leq \frac{1}{1-|c|}\|y\|
$$

Proof. Since $c<0$ and $|c|<\alpha<1$, by Lemma 2.1, we have for $y \in K$ that

$$
\begin{aligned}
\left(A^{-1} y\right)(t) & =y(t)+\sum_{j=1}^{\infty} c^{j} y\left(s-\sum_{i=1}^{j-1} \delta\left(D_{i}\right)\right) \\
& =y(t)+\sum_{j \geq 1 \text { even }} c^{j} y\left(s-\sum_{i=1}^{j-1} \delta\left(D_{i}\right)\right)-\sum_{j \geq 1 \text { odd }}|c|^{j} y\left(s-\sum_{i=1}^{j-1} \delta\left(D_{i}\right)\right) \\
& \geq \alpha\|y\|+\alpha \sum_{j \geq 1 \text { even }} c^{j}\|y\|-\|y\| \sum_{j \geq 1 \text { odd }}|c|^{j} \\
& =\frac{\alpha}{1-c^{2}}\|y\|-\frac{|c|}{1-c^{2}}\|y\| \\
& =\frac{\alpha-|c|}{1-c^{2}}\|y\| .
\end{aligned}
$$

Lemma 2.3. If $c>0$ and $c<1$ then for $y \in K$ one has

$$
\frac{\alpha}{1-c}\|y\| \leq\left(A^{-1} y\right)(t) \leq \frac{1}{1-c}\|y\|
$$


Proof. Since $c>0$ and $c<1, \alpha<1$, by Lemma 2.1, we have for $y \in K$ that

$$
\begin{aligned}
\left(A^{-1} y\right)(t) & =y(t)+\sum_{j \geq 1} c^{j} y\left(s-\sum_{i=1}^{j-1} \delta\left(D_{i}\right)\right) \\
& \geq \alpha\|y\|+\alpha\|y\| \sum_{j \geq 1} c^{j} \\
& =\frac{\alpha}{1-c}\|y\| .
\end{aligned}
$$

\section{Periodic Solutions for Neutral Differential Equation}

In this section, we consider the second-order neutral differential equation

$$
(x(t)-c x(t-\delta(t)))^{\prime \prime}=f\left(t, x^{\prime}(t)\right)+g(t, x(t-\tau(t)))+e(t),
$$

where $\tau, e \in C_{\omega}$ and $\int_{0}^{\omega} e(t) d t=0 ; f$ and $g$ are continuous functions defined on $\mathbb{R}^{2}$ and periodic in $t$ with $f(t, \cdot)=f(t+\omega, \cdot), g(t, \cdot)=g(t+\omega, \cdot), f(t, 0)=0, f(t, u) \geq 0$, or $f(t, u) \leq 0$ for all $(t, u) \in \mathbb{R}^{2}$.

We first recall Mawhin's continuation theorem which our study is based upon. Let $X$ and $Y$ be real Banach spaces and $L: D(L) \subset X \rightarrow Y$ a Fredholm operator with index zero, where $D(L)$ denotes the domain of $L$. This means that $\operatorname{Im} L$ is closed in $Y$ and $\operatorname{dim} \operatorname{Ker} L=$ $\operatorname{dim}(Y / \operatorname{Im} L)<+\infty$. Consider supplementary subspaces $X_{1}, Y_{1}$, of $X, Y$ respectively, such that $X=\operatorname{Ker} L \oplus X_{1}, Y=\operatorname{Im} L \oplus Y_{1}$, and let $P_{1}: X \rightarrow \operatorname{Ker} L$ and $Q_{1}: Y \rightarrow Y_{1}$ denote the natural projections. Clearly, $\operatorname{Ker} L \cap\left(D(L) \cap X_{1}\right)=\{0\}$, thus the restriction $L_{P_{1}}:=\left.L\right|_{D(L) \cap X_{1}}$ is invertible. Let $L_{P_{1}}^{-1}$ denote the inverse of $L_{P_{1}}$.

Let $\Omega$ be an open bounded subset of $X$ with $D(L) \cap \Omega \neq \emptyset$. A map $N: \bar{\Omega} \rightarrow Y$ is said to be $L$-compact in $\bar{\Omega}$ if $Q_{1} N(\bar{\Omega})$ is bounded and the operator $L_{P_{1}}^{-1}\left(I-Q_{1}\right) N: \bar{\Omega} \rightarrow X$ is compact.

Lemma 3.1 (Gaines and Mawhin [8]). Suppose that $X$ and $Y$ are two Banach spaces and $L$ : $D(L) \subset X \rightarrow Y$ is a Fredholm operator with index zero. Furthermore, $\Omega \subset X$ is an open bounded set, and $N: \bar{\Omega} \rightarrow Y$ is L-compact on $\bar{\Omega}$. Assume that the following conditions hold:

(1) $L x \neq \lambda N x$, for all $x \in \partial \Omega \cap D(L), \lambda \in(0,1)$;

(2) $N x \notin \operatorname{Im} L$, for all $x \in \partial \Omega \cap \operatorname{Ker} L$;

(3) $\operatorname{deg}\left\{J Q_{1} N, \Omega \cap \operatorname{Ker} L, 0\right\} \neq 0$, where $J: \operatorname{Im} \quad Q_{1} \rightarrow \operatorname{Ker} L$ is an isomorphism.

Then the equation $L x=N x$ has a solution in $\bar{\Omega} \cap D(L)$.

In order to use Mawhin's continuation theorem to study the existence of $\omega$-periodic solutions for (3.1), we rewrite (3.1) in the following form:

$$
\begin{gathered}
\left(A x_{1}\right)^{\prime}(t)=x_{2}(t), \\
x_{2}^{\prime}(t)=f\left(t, x_{1}^{\prime}(t)\right)+g\left(t, x_{1}(t-\tau(t))\right)+e(t) .
\end{gathered}
$$


Clearly, if $x(t)=\left(x_{1}(t), x_{2}(t)\right)^{\top}$ is an $\omega$-periodic solution to (3.2), then $x_{1}(t)$ must be an $\omega$-periodic solution to (3.1). Thus, the problem of finding an $\omega$-periodic solution for (3.1) reduces to finding one for (3.2).

Recall that $C_{\omega}=\{\phi \in C(\mathbb{R}, \mathbb{R}): \phi(t+\omega) \equiv \phi(t)\}$ with norm $\|\phi\|=\max _{t \in[0, \omega]}|\phi(t)|$. Define $X=Y=C_{\omega} \times C_{\omega}=\left\{x=\left(x_{1}(\cdot), x_{2}(\cdot)\right) \in C\left(\mathbb{R}, \mathbb{R}^{2}\right): x(t)=x(t+\omega), t \in \mathbb{R}\right\}$ with norm $\|x\|=\max \left\{\left\|x_{1}\right\|,\left\|x_{2}\right\|\right\}$. Clearly, $X$ and $Y$ are Banach spaces. Moreover, define

$$
L: D(L)=\left\{x \in C^{1}\left(\mathbb{R}, \mathbb{R}^{2}\right): x(t+\omega)=x(t), t \in \mathbb{R}\right\} \subset X \longrightarrow Y
$$

by

$$
(L x)(t)=\left(\begin{array}{c}
\left(A x_{1}\right)^{\prime}(t) \\
x_{2}^{\prime}(t)
\end{array}\right)
$$

and $N: X \rightarrow Y$ by

$$
(N x)(t)=\left(\begin{array}{c}
x_{2}(t) \\
f\left(t, x_{1}^{\prime}(t)\right)+g\left(t, x_{1}(t-\tau(t))\right)+e(t)
\end{array}\right) .
$$

Then (3.2) can be converted to the abstract equation $L x=N x$. From the definition of $L$, one can easily see that

$$
\operatorname{Ker} L \cong \mathbb{R}^{2}, \quad \operatorname{Im} L=\left\{y \in Y: \int_{0}^{\omega}\left(\begin{array}{l}
y_{1}(s) \\
y_{2}(s)
\end{array}\right) d s=\left(\begin{array}{l}
0 \\
0
\end{array}\right)\right\}
$$

So $L$ is a Fredholm operator with index zero. Let $P_{1}: X \rightarrow \operatorname{Ker} L$ and $Q_{1}: Y \rightarrow \operatorname{Im} Q_{1} \subset \mathbb{R}^{2}$ be defined by

$$
P_{1} x=\left(\begin{array}{c}
\left(A x_{1}\right)(0) \\
x_{2}(0)
\end{array}\right) ; \quad Q_{1} y=\frac{1}{\omega} \int_{0}^{\omega}\left(\begin{array}{l}
y_{1}(s) \\
y_{2}(s)
\end{array}\right) d s,
$$

then $\operatorname{Im} P_{1}=\operatorname{Ker} L, \operatorname{Ker} Q_{1}=\operatorname{Im} L$. Setting $L_{P_{1}}=\left.L\right|_{\mathrm{D}(L) \cap \operatorname{Ker} P_{1}}$ and $L_{P_{1}}^{-1}: \operatorname{Im} L \rightarrow D(L)$ denotes the inverse of $L_{P_{1}}$, then

$$
\begin{gathered}
{\left[L_{P_{1}}^{-1} y\right](t)=\left(\begin{array}{c}
\left(A^{-1} F y_{1}\right)(t) \\
\left(F y_{2}\right)(t)
\end{array}\right),} \\
{\left[F y_{1}\right](t)=\int_{0}^{t} y_{1}(s) d s, \quad\left[F y_{2}\right](t)=\int_{0}^{t} y_{2}(s) d s .}
\end{gathered}
$$

From (3.5) and (3.8), it is clear that $Q_{1} N$ and $L_{P_{1}}^{-1}\left(I-Q_{1}\right) N$ are continuous and $Q_{1} N(\bar{\Omega})$ is bounded, and then $L_{P_{1}}^{-1}\left(I-Q_{1}\right) N(\bar{\Omega})$ is compact for any open bounded $\Omega \subset X$ which means $N$ is $L$-compact on $\bar{\Omega}$. 
Now we give our main results on periodic solutions for (3.1).

Theorem 3.2. Suppose there exist positive constants $K_{1}, D, M, b$ with $M>\|e\|$ such that:

$\left(H_{1}\right)|f(t, u)| \leq K_{1}|u|+b$, for $(t, u) \in \mathbb{R} \times \mathbb{R} ;$

$\left(H_{2}\right) \operatorname{sgn} x \cdot g(t, x)>\|e\|$, for $|x|>D$;

$\left(H_{3}\right) g(t, x) \geq-M$, for $x \leq-D$ and $t \in \mathbb{R}$.

Then (3.1) has at least one solution with period $\omega$ if $0<\omega^{1 / 2}(1+|c|)^{1 / 2} \sqrt{2 K_{1}} /\left(|1-| c||-|c| \delta_{1}\right)<1$, where $\delta_{1}=\max _{t \in[0, \omega]}\left|\delta^{\prime}(t)\right|$.

Proof. By construction (3.2) has an $\omega$-periodic solution if and only if the following operator equation

$$
L x=N x
$$

has an $\omega$-periodic solution. From (3.8), we see that $N$ is $L$-compact on $\bar{\Omega}$, where $\Omega$ is any open, bounded subset of $C_{\omega}$. For $\lambda \in(0,1]$ define

$$
\Omega_{1}=\left\{x \in C_{\omega}: L x=\lambda N x\right\} .
$$

Then $x=\left(x_{1}, x_{2}\right)^{\top} \in \Omega_{1}$ satisfies

$$
\begin{gathered}
\left(A x_{1}\right)^{\prime}(t)=\lambda x_{2}(t), \\
x_{2}^{\prime}(t)=\lambda f\left(t, x_{1}^{\prime}(t)\right)+\lambda g\left(t, x_{1}(t-\tau(t))\right)+\lambda e(t) .
\end{gathered}
$$

We first claim that there is a constant $\xi \in \mathbb{R}$ such that

$$
\left|x_{1}(\xi)\right| \leq D
$$

In view of $\int_{0}^{\omega}\left(A x_{1}\right)^{\prime}(t) d t=0$, we know that there exist two constants $t_{1}, t_{2} \in[0, \omega]$ such that $\left(A x_{1}\right)^{\prime}\left(t_{1}\right) \geq 0,\left(A x_{1}\right)^{\prime}\left(t_{2}\right) \leq 0$. From the first equation of (3.11), we have $x_{2}(t)=$ $(1 / \lambda)\left(A x_{1}\right)^{\prime}(t)$, so

$$
\begin{aligned}
& x_{2}\left(t_{1}\right)=\frac{1}{\lambda}\left(A x_{1}\right)^{\prime}\left(t_{1}\right) \geq 0, \\
& x_{2}\left(t_{2}\right)=\frac{1}{\lambda}\left(A x_{1}\right)^{\prime}\left(t_{2}\right) \leq 0 .
\end{aligned}
$$

Let $t_{3}, t_{4} \in[0, \omega]$ be, respectively, a global maximum and minimum point of $x_{2}(t)$. Clearly, we have

$$
\begin{array}{ll}
x_{2}\left(t_{3}\right) \geq 0, & x_{2}^{\prime}\left(t_{3}\right)=0, \\
x_{2}\left(t_{4}\right) \leq 0, & x_{2}^{\prime}\left(t_{4}\right)=0 .
\end{array}
$$


Since $f\left(t, x_{1}^{\prime}\right) \geq 0$ or $f\left(t, x_{1}^{\prime}\right) \leq 0$, w.l.o.g., suppose $f\left(t, x_{1}^{\prime}\right) \geq 0$, for $\left(t, x_{1}^{\prime}\right) \in[0, \omega] \times \mathbb{R}$. Then

$$
\begin{gathered}
-g\left(t_{3}, x_{1}\left(t_{3}-\tau\left(t_{3}\right)\right)\right)-e\left(t_{3}\right)=f\left(t, x_{1}^{\prime}\left(t_{3}\right)\right) \geq 0, \\
g\left(t_{3}, x_{1}\left(t_{3}-\tau\left(t_{3}\right)\right)\right) \leq-e\left(t_{3}\right) \leq\|e\| .
\end{gathered}
$$

From $\left(H_{2}\right)$ we see that

$$
x_{1}\left(t_{3}-\tau\left(t_{3}\right)\right)<D \text {. }
$$

Similarly, we have

$$
g\left(t_{4}, x_{1}\left(t_{4}-\tau\left(t_{4}\right)\right)\right) \geq-e\left(t_{4}\right) \geq-\|e\|,
$$

and again by $\left(H_{2}\right)$,

$$
x_{1}\left(t_{4}-\tau\left(t_{4}\right)\right)<-D \text {. }
$$

Case 1. If $x_{1}\left(t_{3}-\tau\left(t_{3}\right)\right) \in(-D, D)$, define $\xi=t_{3}-\tau\left(t_{3}\right)$, obviously $\left|x_{1}(\xi)\right| \leq D$.

Case 2. If $x_{1}\left(t_{3}-\tau\left(t_{3}\right)\right)<-D$, from (3.18) and the fact that $x$ is a continuous function in $\mathbb{R}$, there exists a constant $\xi$ between $x_{1}\left(t_{3}-\tau\left(t_{3}\right)\right)$ and $x_{1}\left(t_{4}-\tau\left(t_{4}\right)\right)$ such that $\left|x_{1}(\xi)\right|=D$. This proves (3.12).

Choose an integer $k$ and a constant $t_{5} \in[0, \omega]$ such that $\xi=\omega k+t_{5}$, then $\left|x_{1}(\xi)\right|=$ $\left|x_{1}\left(t_{5}\right)\right| \leq D$. Hence

$$
\left|x_{1}(t)\right| \leq D+\int_{0}^{\omega}\left|x_{1}^{\prime}(s)\right| d s
$$

Substituting $x_{2}(t)=(1 / \lambda)\left(A x_{1}\right)^{\prime}(t)$ into the second equation of (3.11) yields

$$
\left(\frac{1}{\lambda}\left(A x_{1}\right)(t)\right)^{\prime \prime}=\lambda f\left(t, x_{1}^{\prime}(t)\right)+\lambda g\left(t, x_{1}(t-\tau(t))\right)+\lambda e(t)
$$

that is,

$$
\left(\left(A x_{1}\right)(t)\right)^{\prime \prime}=\lambda^{2} f\left(t, x_{1}^{\prime}(t)\right)+\lambda^{2} g\left(t, x_{1}(t-\tau(t))\right)+\lambda^{2} e(t) .
$$

Integrating both sides of $(3.21)$ over $[0, \omega]$, we have

$$
\int_{0}^{\omega}\left[f\left(t, x_{1}^{\prime}(t)\right)+g\left(t, x_{1}(t-\tau(t))\right)\right] d t=0
$$


On the other hand, multiplying both sides of $(3.21)$ by $\left(A x_{1}\right)(t)$ and integrating over $[0, \omega]$, we get

$$
\begin{aligned}
\int_{0}^{\omega}\left(\left(A x_{1}\right)(t)\right)^{\prime \prime}\left(A x_{1}(t)\right) d t= & -\int_{0}^{\omega}\left|\left(A x_{1}\right)^{\prime}(t)\right|^{2} d t=-\lambda^{2} \int_{0}^{\omega} f\left(t, x_{1}^{\prime}(t)\right)\left(A x_{1}\right)(t) d t \\
& -\lambda^{2} \int_{0}^{\omega} g\left(t, x_{1}(t-\tau(t))\right)\left(A x_{1}\right)(t) d t-\lambda^{2} \int_{0}^{\omega} e(t)\left(A x_{1}\right)(t) d t .
\end{aligned}
$$

Using $\left(H_{1}\right)$, we have

$$
\begin{aligned}
\int_{0}^{\omega}\left|\left(A x_{1}\right)^{\prime}(t)\right|^{2} d t \leq & \int_{0}^{\omega}\left|f\left(t, x_{1}^{\prime}(t)\right)\right|\left|\left[x_{1}(t)-c x_{1}(t-\delta(t))\right]\right| d t \\
& +\int_{0}^{\omega}\left|g\left(t, x_{1}(t-\tau(t))\right)\right|\left|\left[x_{1}(t)-c x_{1}(t-\delta(t))\right]\right| d t \\
& +\int_{0}^{\omega}\left|e(t) \|\left[x_{1}(t)-c x_{1}(t-\delta(t))\right]\right| d t \\
\leq & (1+|c|)\left\|x_{1}\right\|\left[K_{1} \int_{0}^{\omega}\left|x_{1}^{\prime}(t)\right| d t+b \omega+\int_{0}^{\omega}\left|g\left(t, x_{1}(t-\tau(t))\right)\right| d t+\omega\|e\|\right]
\end{aligned}
$$

Besides, we can assert that there exists some positive constant $N_{1}$ such that

$$
\int_{0}^{\omega}\left|g\left(t, x_{1}(t-\tau(t))\right)\right| d t \leq 2 \omega N_{1}+\omega b+K_{1} \int_{0}^{\omega}\left|x_{1}^{\prime}(t)\right| d t
$$

In fact, in view of condition $\left(H_{1}\right)$ and (3.22) we have

$$
\begin{aligned}
\int_{0}^{\omega}\left\{g\left(t, x_{1}(t-\tau(t))\right)-K_{1}\left|x_{1}^{\prime}(t)\right|-b\right\} d t & \leq \int_{0}^{\omega}\left\{g\left(t, x_{1}(t-\tau(t))\right)-\left|f\left(t, x_{1}^{\prime}(t)\right)\right|\right\} d t \\
& \leq \int_{0}^{\omega}\left\{g\left(t, x_{1}(t-\tau(t))\right)+f\left(t, x_{1}^{\prime}(t)\right)\right\} d t \\
& =0 .
\end{aligned}
$$

\section{Define}

$$
\begin{aligned}
& E_{1}=\left\{t \in[0, \omega]: x_{1}(t-\tau(t))>D\right\} \\
& E_{2}=\left\{t \in[0, \omega]:\left|x_{1}(t-\tau(t))\right| \leq D\right\} \cup\left\{t \in[0, \omega]: x_{1}(t-\tau(t))<-D\right\}
\end{aligned}
$$


With these sets we get

$$
\begin{gathered}
\int_{E_{2}}\left|g\left(t, x_{1}(t-\tau(t))\right)\right| d t \leq \omega \max \left\{M, \sup _{t \in[0, \omega], x_{1}(t-\tau(t)) \mid \leq D}\left|g\left(t, x_{1}\right)\right|\right\} \\
\int_{E_{1}}\left\{\left|g\left(t, x_{1}(t-\tau(t))\right)\right|-K_{1}\left|x_{1}^{\prime}(t)\right|-b\right\} d t \\
\quad \int_{E_{1}}\left\{g\left(t, x_{1}(t-\tau(t))\right)-K_{1}\left|x_{1}^{\prime}(t)\right|-b\right\} d t \\
\leq-\int_{E_{2}}\left\{g\left(t, x_{1}(t-\tau(t))\right)-K_{1}\left|x_{1}^{\prime}(t)\right|-b\right\} d t \\
\leq \int_{E_{2}}\left\{\left|g\left(t, x_{1}(t-\tau(t))\right)\right|+K_{1}\left|x_{1}^{\prime}(t)\right|+b\right\} d t
\end{gathered}
$$

which yields

$$
\begin{aligned}
\int_{E_{1}}\left|g\left(t, x_{1}(t-\tau(t))\right)\right| d t & \leq \int_{E_{2}}\left|g\left(t, x_{1}(t-\tau(t))\right)\right| d t+\int_{E_{1} \cup E_{2}}\left(K_{1}\left|x_{1}^{\prime}(t)\right|+b\right) d t \\
& =\int_{E_{2}}\left|g\left(t, x_{1}(t-\tau(t))\right)\right| d t+\omega b+K_{1} \int_{0}^{\omega}\left|x_{1}^{\prime}(t)\right| d t .
\end{aligned}
$$

That is,

$$
\begin{aligned}
\int_{0}^{\omega}\left|g\left(t, x_{1}(t-\tau(t))\right)\right| d t & =\int_{E_{1}}\left|g\left(t, x_{1}(t-\tau(t))\right)\right| d t+\int_{E_{2}}\left|g\left(x_{1}(t-\tau(t))\right)\right| d t \\
& \leq 2 \int_{E_{2}}\left|g\left(t, x_{1}(t-\tau(t))\right)\right| d t+\omega b+K_{1} \int_{0}^{\omega}\left|x_{1}^{\prime}(t)\right| d t \\
& \leq 2 \omega \max \left\{M_{t \in[0, \omega],\left|x_{1}(t-\tau(t))\right|<D}\left|g\left(t, x_{1}\right)\right|\right\}+\omega b+K_{1} \int_{0}^{\omega}\left|x_{1}^{\prime}(t)\right| d t \\
& =2 \omega D_{1}+\omega b+K_{1} \int_{0}^{\omega}\left|x_{1}^{\prime}(t)\right| d t
\end{aligned}
$$

where $N_{1}=\max \left\{M, \sup _{t \in[0, \omega],\left|x_{1}(t-\tau(t))\right|<D}\left|g\left(t, x_{1}\right)\right|\right\}$, proving (3.25). 
Substituting (3.25) into (3.24) and recalling (3.19), we get

$$
\begin{gathered}
\int_{0}^{\omega}\left|\left(A x_{1}\right)^{\prime}(t)\right|^{2} d t \leq(1+|c|)\left|x_{1}\right|_{0}\left(2 K_{1} \int_{0}^{\omega}\left|x_{1}^{\prime}(t)\right| d t+2 \omega b+2 \omega N_{1}+\omega \max _{t \in[0, \omega]}|e(t)|\right) \\
=(1+|c|)\left(2 K_{1}\left|x_{1}\right|_{0} \int_{0}^{\omega}\left|x_{1}^{\prime}(t)\right| d t+2 \omega b\left|x_{1}\right|_{0}+2 \omega N_{1}\left|x_{1}\right|_{0}+\omega\left|x_{1}\right|_{0} \max _{t \in[0, \omega]}|e(t)|\right) \\
\leq(1+|c|)\left[2 K_{1}\left(D+\int_{0}^{\omega}\left|x_{1}^{\prime}(t)\right| d t\right) \int_{0}^{\omega}\left|x_{1}^{\prime}(t)\right| d t\right. \\
\left.\quad+\left(2 \omega b+2 \omega N_{1}+\omega \max _{t \in[0, \omega]}|e(t)|\right)\left(D+\int_{0}^{\omega}\left|x_{1}^{\prime}(t)\right| d t\right)\right] \\
=(1+|c|)\left[2 K_{1} D \int_{0}^{\omega}\left|x_{1}^{\prime}(t)\right| d t+2 K_{1}\left(\int_{0}^{\omega}\left|x_{1}^{\prime}(t)\right| d t\right)^{2}+N_{2} \int_{0}^{\omega}\left|x_{1}^{\prime}(t)\right| d t+N_{2} D\right] \\
=2 K_{1}(1+|c|)\left(\int_{0}^{\omega}\left|x_{1}^{\prime}(t)\right| d t\right)^{2}+(1+|c|)\left(N_{2}+2 K_{1} D\right) \int_{0}^{\omega}\left|x_{1}^{\prime}(t)\right| d t+(1+|c|) N_{2} D,
\end{gathered}
$$

where $N_{2}=2 \omega b+2 \omega N_{1}+\omega\|e\|$. Since $(A x)(t)=x(t)-c x(t-\delta(t))$, we have

$$
\begin{aligned}
\left(A x_{1}\right)^{\prime}(t) & =\left(x_{1}(t)-c x_{1}(t-\delta(t))\right)^{\prime} \\
& =x_{1}^{\prime}(t)-c x_{1}^{\prime}(t-\delta(t))+c x_{1}^{\prime}(t-\delta(t)) \delta^{\prime}(t) \\
& =\left(A x_{1}^{\prime}\right)(t)+c x_{1}^{\prime}(t-\delta(t)) \delta^{\prime}(t), \\
\left(A x_{1}^{\prime}\right)(t) & =\left(A x_{1}\right)^{\prime}(t)-c x_{1}^{\prime}(t-\delta(t)) \delta^{\prime}(t) .
\end{aligned}
$$

By applying Lemma 2.1, we have

$$
\begin{aligned}
\int_{0}^{\omega}\left|x_{1}^{\prime}(t)\right| d t & =\int_{0}^{\omega}\left|\left(A^{-1} A x_{1}^{\prime}\right)(t)\right| d t \\
& \leq \frac{\int_{0}^{\omega}\left|\left(A x_{1}^{\prime}\right)(t)\right| d t}{|1-| c||} \\
& =\frac{\int_{0}^{\omega}\left|\left(A x_{1}\right)^{\prime}(t)-c x_{1}^{\prime}(t-\delta(t)) \delta^{\prime}(t)\right| d t}{|1-| c||} \\
& \leq \frac{\int_{0}^{\omega}\left|\left(A x_{1}^{\prime}\right)(t)\right| d t+|c| \delta_{1} \int_{0}^{\omega}\left|x_{1}^{\prime}(t)\right| d t}{|1-| c||}
\end{aligned}
$$


where $\delta_{1}=\max _{t \in[0, \omega]}\left|\delta^{\prime}(t)\right|$. Since $0<\omega^{1 / 2}(1+|c|)^{1 / 2} \sqrt{2 K_{1}} /\left(|1-| c||-|c| \delta_{1}\right)$, then $|1-| c||-|c| \delta_{1}>$ 0 , so we get

$$
\int_{0}^{\omega}\left|x_{1}^{\prime}(t)\right| d t \leq \frac{\int_{0}^{\omega}\left|\left(A x_{1}\right)^{\prime}(t)\right| d t}{|1-| c||-|c| \delta_{1}} \leq \frac{\omega^{1 / 2}\left(\int_{0}^{\omega}\left|\left(A x_{1}\right)^{\prime}(t)\right|^{2} d t\right)^{1 / 2}}{|1-| c||-|c| \delta_{1}} .
$$

Applying the inequality $(a+b)^{k} \leq a^{k}+b^{k}$ for $a, b>0,0<k<1$, it follows from (3.31) and (3.34) that

$$
\begin{aligned}
& \int_{0}^{\omega}\left|x_{1}^{\prime}(t)\right| d t \\
& \leq \frac{\omega^{1 / 2}}{|1-| c||-|c| \delta_{1}}\left[(1+|c|)^{1 / 2} \sqrt{2 K_{1}} \int_{0}^{\omega}\left|x_{1}^{\prime}(t)\right| d t+(1+|c|)^{1 / 2}\left(\int_{0}^{\omega}\left|x_{1}^{\prime}(t)\right| d t\right)^{1 / 2}\right. \\
& \left.\times\left(N_{2}+2 K_{1} D\right)^{1 / 2}+(1+|c|)^{1 / 2} N_{2} D^{1 / 2}\right] .
\end{aligned}
$$

Since $\omega^{1 / 2}(1+|c|)^{1 / 2} \sqrt{2 K_{1}} /\left(|1-| c||-|c| \delta_{1}\right)<1$, it is easy to see that there exists a constant $M_{1}>0$ (independent of $\lambda$ ) such that

$$
\int_{0}^{\omega}\left|x_{1}^{\prime}(t)\right| d t \leq M_{1}
$$

It follows from (3.19) that

$$
\left\|x_{1}\right\| \leq D+\int_{0}^{\omega}\left|x_{1}^{\prime}(t)\right| d t \leq D+M_{1}:=M_{2}
$$

By the first equation of (3.11) we have $\int_{0}^{\omega} x_{2}(t) d t=\int_{0}^{\omega}\left(A x_{1}\right)^{\prime}(t) d t=0$, which implies that there is a constant $t_{1} \in[0, \omega]$ such that $x_{2}\left(t_{1}\right)=0$, hence $\left\|x_{2}\right\| \leq \int_{0}^{\omega}\left|x_{2}^{\prime}(t)\right| d t$. By the second equation of (3.11) we obtain

$$
x_{2}^{\prime}(t)=\lambda f\left(t, x_{1}^{\prime}(t)\right)+\lambda g\left(x_{1}(t-\tau(t))\right)+\lambda e(t) .
$$

So, from $\left(H_{1}\right)$ and (3.25), we have

$$
\begin{aligned}
\left|x_{2}\right|_{0} & \leq \int_{0}^{\omega}\left|f\left(t, x_{1}^{\prime}(t)\right)\right| d t+\int_{0}^{\omega}\left|g\left(t, x_{1}(t-\tau(t))\right)\right| d t+\int_{0}^{\omega}|e(t)| d t \\
& \leq 2 K_{1} M_{1}+2 \omega b+2 \omega N_{1}+\omega\|e\|:=M_{3} .
\end{aligned}
$$


Let $M_{4}=\sqrt{M_{2}^{2}+M_{3}^{2}}+1, \Omega=\left\{x=\left(x_{1}, x_{2}\right)^{\top}:\left\|x_{1}\right\|<M_{4},\left\|x_{2}\right\|<M_{4}\right\}$, then for all $x \in$ $\partial \Omega \cap \operatorname{Ker} L$

$$
Q_{1} N x=\frac{1}{\omega} \int_{0}^{\omega}\left(\begin{array}{c}
x_{2}(t) \\
f\left(t, x_{1}^{\prime}(t)\right)+g\left(t, x_{1}(t-\tau(t))\right)+e(t)
\end{array}\right) d t
$$

If $Q_{1} N x=0$, then $x_{2}(t)=0, x_{1}=M_{4}$ or $-M_{4}$. But if $x_{1}(t)=M_{4}$, we know

$$
0=\int_{0}^{\omega} g\left(M_{4}\right) d t
$$

that is, $g\left(M_{4}\right)=0$. From assumption $\left(H_{2}\right)$, we know $M_{4} \leq D$, which yields a contradiction, one can argue similarly if $x_{1}=-M_{4}$. We also have $Q_{1} N x \neq 0$, that is, for all $x \in \partial \Omega \cap \operatorname{Ker} L, x \notin$ $\operatorname{Im} L$, so conditions (1) and (2) of Lemma 3.1 are both satisfied. Define the isomorphism $J$ : $\operatorname{Im} Q_{1} \rightarrow \operatorname{Ker} L$ as follows:

$$
J\left(x_{1}, x_{2}\right)^{\top}=\left(x_{2}, x_{1}\right)^{\top}
$$

Let $H(\mu, x)=\mu x+(1-\mu) J Q_{1} N x,(\mu, x) \in[0,1] \times \Omega$, then, for all $(\mu, x) \in(0,1) \times(\partial \Omega \cap \operatorname{Ker} L)$,

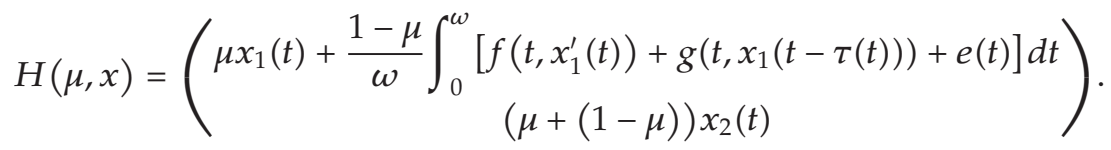

We have $\int_{0}^{\omega} e(t) d t=0$. So, we can get

$$
\begin{aligned}
& H(\mu, x)=\left(\begin{array}{c}
\mu x_{1}(t)+\frac{1-\mu}{\omega} \int_{0}^{\omega}\left[f\left(t, x_{1}^{\prime}(t)\right)+g\left(t, x_{1}(t-\tau(t))\right)\right] d t \\
(\mu+(1-\mu)) x_{2}(t)
\end{array}\right), \\
& \forall(\mu, x) \in(0,1) \times(\partial \Omega \cap \operatorname{Ker} L) .
\end{aligned}
$$

From $\left(H_{2}\right)$, it is obvious that $x^{\top} H(\mu, x)>0$, for all $(\mu, x) \in(0,1) \times(\partial \Omega \cap \operatorname{Ker} L)$. Hence

$$
\begin{aligned}
\operatorname{deg}\left\{J Q_{1} N, \Omega \cap \operatorname{Ker} L, 0\right\} & =\operatorname{deg}\{H(0, x), \Omega \cap \operatorname{Ker} L, 0\} \\
& =\operatorname{deg}\{H(1, x), \Omega \cap \operatorname{Ker} L, 0\} \\
& =\operatorname{deg}\{I, \Omega \cap \operatorname{Ker} L, 0\} \neq 0 .
\end{aligned}
$$

So condition (3) of Lemma 3.1 is satisfied. By applying Lemma 3.1, we conclude that equation $L x=N x$ has a solution $x=\left(x_{1}, x_{2}\right)^{\top}$ on $\bar{\Omega} \cap D(L)$, that is, (3.1) has an $\omega$-periodic solution $x_{1}(t)$. 
By using a similar argument, we can obtain the following theorem.

Theorem 3.3. Suppose there exist positive constants $K_{1}, D, M, b$ with $M>\|e\|$ such that:

$\left(\mathrm{H}_{1}\right)|f(t, u)| \leq K_{1}|u|+b$, for $(t, u) \in \mathbb{R} \times \mathbb{R} ;$

$\left(\mathrm{H}_{2}\right) \operatorname{sgn} x \cdot g(t, x)>\|e\|$, for $|x|>D$,

$\left(\mathrm{H}_{3}\right) g(t, x) \leq M$, for $x \geq D$ and $t \in \mathbb{R}$,

then (3.1) has at least one solution with period $\omega$ if $0<\omega(1+|c|)^{1 / 2} \sqrt{2 K_{1}} /\left(|1-| c||-|c| \delta_{1}\right)<1$.

Remark 3.4. If $\int_{0}^{\omega} e(t) d t \neq 0$ and $f(t, 0) \neq 0$, the problem of existence of $\omega$-periodic solutions to (3.1) can be converted to the existence of $\omega$-periodic solutions to

$$
(x(t)-c x(t-\delta(t)))^{\prime \prime}=f_{1}\left(t, x^{\prime}(t)\right)+g_{1}(t, x(t-\tau(t)))+e_{1}(t),
$$

where $f_{1}(t, x)=f(t, x)-f(t, 0), g_{1}(t, x)=g(t, x)+(1 / \omega) \int_{0}^{\omega} e(t) d t+f(t, 0)$, and $e_{1}(t)=e(t)-$ $(1 / \omega) \int_{0}^{\omega} e(t) d t$. Clearly, $\int_{0}^{\omega} e_{1}(t) d t=0$ and $f_{1}(t, 0)=0$, and (3.46) can be discussed by using Theorem 3.2 (or Theorem 3.3).

\section{Positive Periodic Solutions for Neutral Equations}

Consider the following second-order neutral functional differential equation:

$$
(x(t)-c x(t-\delta(t)))^{\prime \prime}=-a(t) x(t)+\lambda b(t) f(x(t-\tau(t))),
$$

where $\lambda$ is a positive parameter; $f \in C(\mathbb{R},[0, \infty))$, and $f(x)>0$ for $x>0 ; a \in C(\mathbb{R},(0, \infty))$ with $\max \{a(t): t \in[0, \omega]\}<(\pi / \omega)^{2}, b \in C(\mathbb{R},(0, \infty)), \tau \in C(\mathbb{R}, \mathbb{R}), a(t), b(t)$, and $\tau(t)$ are $\omega$-periodic functions.

Define the Banach space $X$ as in Section 2, and let $C_{\omega}^{+}=\{x \in C(\mathbb{R},(0, \infty)): x(t+\omega)=$ $x(t)\}$. Denote

$$
\begin{gathered}
M=\max \{a(t): t \in[0, \omega]\}, \quad m=\min \{a(t): t \in[0, \omega]\}, \quad \beta=\sqrt{M}, \\
L=\frac{1}{2 \beta \sin (\beta \omega / 2)}, \quad l=\frac{\cos (\beta \omega / 2)}{2 \beta \sin (\beta \omega / 2)}, \quad k=l(M+m)+L M, \\
k_{1}=\frac{k-\sqrt{k^{2}-4 L l M m}}{2 L M}, \quad \alpha=\frac{l[m-(M+m)|c|]}{L M(1-|c|)} .
\end{gathered}
$$

It is easy to see that $M, m, \beta, L, l, k, k_{1}>0$.

Now we consider (4.1). First let

$$
\bar{f}_{0}=\varlimsup_{x \rightarrow 0} \frac{f(x)}{x}, \quad \bar{f}_{\infty}=\varlimsup_{x \rightarrow \infty} \frac{f(x)}{x}, \quad \underline{f}_{0}=\varliminf_{x \rightarrow 0} \frac{f(x)}{x}, \quad \underline{f}_{\infty}=\varliminf_{x \rightarrow \infty} \frac{f(x)}{x},
$$


and denote

$$
\begin{array}{cl}
\bar{i}_{0}=\text { number of } 0^{\prime} \sin \left(\bar{f}_{0}, \bar{f}_{\infty}\right), & \underline{i}_{0}=\text { number of } 0^{\prime} \sin \left(\underline{f}_{0^{\prime}} \underline{f} \underline{-}_{\infty}\right) ; \\
\bar{i}_{\infty}=\text { number of } \infty \text { 's } \operatorname{in}\left(\bar{f}_{0}, \bar{f}_{\infty}\right), & \underline{i}_{\infty}=\text { number of } \infty \text { 's } \operatorname{in}\left(\underline{f}_{0^{\prime}} \underline{f}_{-\infty}\right) .
\end{array}
$$

It is clear that $\bar{i}_{0}, \underline{i}_{0}, \bar{i}_{\infty}, \underline{i}_{\infty} \in\{0,1,2\}$. We will show that (4.1) has $\bar{i}_{0}$ or $\underline{i}_{\infty}$ positive $w$-periodic solutions for sufficiently large or small $\lambda$, respectively.

In the following we discuss (4.1) in two cases, namely, the case where $c<0$ and $c\rangle$ $-\min \left\{k_{1}, m /(M+m)\right\}$ (note that $c>-m /(M+m)$ implies $\alpha>0 ; c>-k_{1}$ implies $|c|<\alpha$ ) and the case where $c>0$ and $c<\min \{m /(M+m),(L M-l m) /((L-l) M-l m)\}$ (note that $c<m /(M+m)$ implies $\alpha>0 ; c<(L M-l m) /((L-l) M-l m)$ implies $\alpha<1)$. Obviously, we have $|c|<1$ which makes Lemma 2.1 applicable for both cases and also Lemmas 2.2 or 2.3, respectively.

Let $K=\{x \in X: x(t) \geq \alpha\|x\|\}$ denote the cone in $X$ as defined in Section 2, where $\alpha$ is just as defined above. We also use $K_{r}=\{x \in K:\|x\|<r\}$ and $\partial K_{r}=\{x \in K:\|x\|=r\}$.

Let $y(t)=(A x)(t)$, then from Lemma 2.1 we have $x(t)=\left(A^{-1} y\right)(t)$. Hence (4.1) can be transformed into

$$
y^{\prime \prime}(t)+a(t)\left(A^{-1} y\right)(t)=\lambda b(t) f\left(\left(A^{-1} y\right)(t-\tau(t))\right)
$$

which can be further rewritten as

$$
y^{\prime \prime}(t)+a(t) y(t)-a(t) H(y(t))=\lambda b(t) f\left(\left(A^{-1} y\right)(t-\tau(t))\right)
$$

where $H(y(t))=y(t)-\left(A^{-1} y\right)(t)=-c\left(A^{-1} y\right)(t-\delta(t))$.

Now we discuss the two cases separately.

\subsection{Case I}

Assume $c<0$ and $c>-\min \left\{k_{1}, m /(M+m)\right\}$.

Lemma 4.1 (see [7]). The equation

$$
y^{\prime \prime}(t)+M y(t)=h(t), \quad h \in C_{\omega \prime}^{+}
$$

has a unique w-periodic solution

$$
y(t)=\int_{t}^{t+\omega} G(t, s) h(s) d s,
$$

where

$$
G(t, s)=\frac{\cos \beta((\omega / 2)+t-s)}{2 \beta \sin (\beta \omega / 2)}, \quad s \in[t, t+\omega] .
$$


Lemma 4.2 (see [7]). One has $\int_{t}^{t+\omega} G(t, s) d s=1 / M$. Furthermore, if $\max \{a(t): t \in[0, \omega]\}<$ $(\pi / \omega)^{2}$, then $0<l \leq G(t, s) \leq L$ for all $t \in[0, \omega]$ and $s \in[t, t+\omega]$.

Now we consider

$$
y^{\prime \prime}(t)+a(t) y(t)-a(t) H(y(t))=h(t), \quad h \in C_{\omega}^{+}
$$

and define operators $T, \widehat{H}: X \rightarrow X$ by

$$
(T h)(t)=\int_{t}^{t+\omega} G(t, s) h(s) d s, \quad(\widehat{H} y)(t)=M-a(t) y(t)+a(t) H(y(t)) .
$$

Clearly $T, \widehat{H}$ are completely continuous $(T h)(t)>0$ for $h(t)>0$ and $\|\widehat{H}\| \leq(M-m+M(|c| /(1-$ $|c|))$ ).

By Lemma 4.1, the solution of (4.10) can be written in the form

$$
y(t)=(T h)(t)+(T \widehat{H} y)(t)
$$

In view of $c<0$ and $c>-\min \left\{k_{1}, m /(M+m)\right\}$, we have

$$
\|T \widehat{H}\| \leq\|T\|\|\widehat{H}\| \leq \frac{M-m+m|c|}{M(1-|c|)}<1
$$

and hence

$$
y(t)=(I-T \widehat{H})^{-1}(T h)(t) .
$$

Define an operator $P: X \rightarrow X$ by

$$
(P h)(t)=(I-T \widehat{H})^{-1}(T h)(t) .
$$

Obviously, for any $h \in C_{\omega}^{+}$, if $\max \{a(t): t \in[0, \omega]\}<(\pi / \omega)^{2}, y(t)=(P h)(t)$ is the unique positive $\omega$-periodic solution of (4.10).

Lemma 4.3. $P$ is completely continuous and

$$
(T h)(t) \leq(P h)(t) \leq \frac{M(1-|c|)}{m-(M+m)|c|}\|T h\|, \quad \forall h \in C_{\omega}^{+} .
$$


Proof. By the Neumann expansion of $P$, we have

$$
\begin{aligned}
P & =(I-T \widehat{H})^{-1} T \\
& =\left(I+T \widehat{H}+(T \widehat{H})^{2}+\cdots+(T \widehat{H})^{n}+\cdots\right) T \\
& =T+T \widehat{H} T+(T \widehat{H})^{2} T+\cdots+(T \widehat{H})^{n} T+\cdots
\end{aligned}
$$

Since $T$ and $\widehat{H}$ are completely continuous, so is $P$. Moreover, by (4.17), and recalling that $\|T \widehat{H}\| \leq(M-m+m|c|) / M(1-|c|)<1$, we get

$$
(T h)(t) \leq(P h)(t) \leq \frac{M(1-|c|)}{m-(M+m)|c|}\|T h\| .
$$

Define an operator $Q: X \rightarrow X$ by

$$
Q y(t)=P\left(\lambda b(t) f\left(\left(A^{-1} y\right)(t-\tau(t))\right)\right)
$$

Lemma 4.4. One has $Q(K) \subset K$.

Proof. From the definition of $Q$, it is easy to verify that $Q y(t+\omega)=Q y(t)$. For $y \in K$, we have from Lemma 4.3 that

$$
\begin{aligned}
Q y(t) & =P\left(\lambda b(t) f\left(\left(A^{-1} y\right)(t-\tau(t))\right)\right) \\
& \geq T\left(\lambda b(t) f\left(\left(A^{-1} y\right)(t-\tau(t))\right)\right) \\
& =\lambda \int_{t}^{t+\omega} G(t, s) b(s) f\left[\left(A^{-1} y\right)(s-\tau(s))\right] d s \\
& \geq \lambda l \int_{0}^{\omega} b(s) f\left[\left(A^{-1} y\right)(s-\tau(s))\right] d s .
\end{aligned}
$$

On the other hand,

$$
\begin{aligned}
Q y(t) & =P\left(\lambda b(t) f\left(\left(A^{-1} y\right)(t-\tau(t))\right)\right) \\
& \leq \frac{M(1-|c|)}{m-(M+m)|c|}\left\|T\left(\lambda b(t) f\left(\left(A^{-1} y\right)(t-\tau(t))\right)\right)\right\|
\end{aligned}
$$




$$
\begin{aligned}
& =\lambda \frac{M(1-|c|)}{m-(M+m)|c|} \max _{t \in[0, \omega]} \int_{t}^{t+\omega} G(t, s) b(s) f\left(\left(A^{-1} y\right)(s-\tau(s))\right) d s \\
& \leq \lambda \frac{M(1-|c|)}{m-(M+m)|c|} L \int_{0}^{\omega} b(s) f\left(\left(A^{-1} y\right)(s-\tau(s))\right) d s .
\end{aligned}
$$

Therefore,

$$
Q y(t) \geq \frac{l[m-(M+m)|c|]}{L M(1-|c|)}\|Q y\|=\alpha\|Q y\|
$$

that is, $Q(K) \subset K$.

From the continuity of $P$, it is easy to verify that $Q$ is completely continuous in $X$. Comparing (4.6) to (4.10), it is obvious that the existence of periodic solutions for (4.6) is equivalent to the existence of fixed points for the operator $Q$ in $X$. Recalling Lemma 4.4, the existence of positive periodic solutions for (4.6) is equivalent to the existence of fixed points of $Q$ in $K$. Furthermore, if $Q$ has a fixed point $y$ in $K$, it means that $\left(A^{-1} y\right)(t)$ is a positive $\omega$-periodic solutions of (4.1).

Lemma 4.5. If there exists $\eta>0$ such that

$$
f\left(\left(A^{-1} y\right)(t-\tau(t))\right) \geq\left(A^{-1} y\right)(t-\tau(t)) \eta, \quad \text { for } t \in[0, \omega], y \in K
$$

then

$$
\|Q y\| \geq \lambda \ln \frac{\alpha-|c|}{1-c^{2}} \int_{0}^{\omega} b(s) d s\|y\|, \quad y \in K
$$

Proof. By Lemmas 2.2, 4.2, and 4.3, we have for $y \in K$ that

$$
\begin{aligned}
Q y(t) & =P\left(\lambda b(t) f\left(\left(A^{-1} y\right)(t-\tau(t))\right)\right) \\
& \geq T\left(\lambda b(t) f\left(\left(A^{-1} y\right)(t-\tau(t))\right)\right) \\
& =\lambda \int_{t}^{t+\omega} G(t, s) b(s) f\left(\left(A^{-1} y\right)(s-\tau(s))\right) d s \\
& \geq \lambda l \eta \int_{0}^{\omega} b(s)\left(A^{-1} y\right)(s-\tau(s)) d s \\
& \geq \lambda l \eta \frac{\alpha-|c|}{1-c^{2}} \int_{0}^{\omega} b(s) d s\|y\| .
\end{aligned}
$$


Hence

$$
\|Q y\| \geq \lambda \ln \frac{\alpha-|c|}{1-c^{2}} \int_{0}^{\omega} b(s) d s\|y\|, \quad y \in K
$$

Lemma 4.6. If there exists $\varepsilon>0$ such that

$$
f\left(\left(A^{-1} y\right)(t-\tau(t))\right) \leq\left(A^{-1} y\right)(t-\tau(t)) \varepsilon, \quad \text { for } t \in[0, \omega], y \in K,
$$

then

$$
\|Q y\| \leq \lambda \varepsilon \frac{L M \int_{0}^{\omega} b(s) d s}{m-(M+m)|c|}\|y\|, \quad y \in K .
$$

Proof. By Lemmas 2.2, 4.2, and 4.3, we have

$$
\begin{aligned}
\|Q y(t)\| & \leq \lambda \frac{M(1-|c|)}{m-(M+m)|c|} L \int_{0}^{\omega} b(s) f\left(\left(A^{-1} y\right)(s-\tau(s))\right) d s \\
& \leq \lambda \frac{M(1-|c|)}{m-(M+m)|c|} L \varepsilon \int_{0}^{\omega} b(s)\left(A^{-1} y\right)(s-\tau(s)) d s \\
& \leq \lambda \varepsilon \frac{L M \int_{0}^{\omega} b(s) d s}{m-(M+m)|c|}\|y\| .
\end{aligned}
$$

Define

$$
\begin{aligned}
& F(r)=\max \left\{f(t): 0 \leq t \leq \frac{r}{1-|c|}\right\} \\
& f_{1}(r)=\min \left\{f(t): \frac{\alpha-|c|}{1-c^{2}} r \leq t \leq \frac{r}{1-|c|}\right\} .
\end{aligned}
$$

Lemma 4.7. If $y \in \partial K_{r}$, then

$$
\|Q y\| \geq \lambda l f_{1}(r) \int_{0}^{\omega} b(s) d s
$$

Proof. By Lemma 2.2, we obtain $\left((\alpha-|c|) /\left(1-c^{2}\right)\right) r \leq\left(A^{-1} y\right)(t-\tau(t)) \leq r /(1-|c|)$ for $y \in \partial K_{r}$, which yields $f\left(\left(A^{-1} y\right)(t-\tau(t))\right) \geq f_{1}(r)$. The lemma now follows analog to the proof of Lemma 4.5. 
Lemma 4.8. If $y \in \partial K_{r}$, then

$$
\|Q y\| \leq \lambda \frac{\operatorname{LM}(1-|c|) F(r)}{m-(M+m)|c|} \int_{0}^{\omega} b(s) d s
$$

Proof. By Lemma 2.2, we can have $0 \leq\left(A^{-1} y\right)(t-\tau(t)) \leq r /(1-|c|)$ for $y \in \partial K_{r}$, which yields $f\left(\left(A^{-1} y\right)(t-\tau(t))\right) \leq F(r)$. Similar to the proof of Lemma 4.6, we get the conclusion.

We quote the fixed point theorem which our results will be based on.

Lemma 4.9 (see [9]). Let $X$ be a Banach space and $K$ a cone in $X$. For $r>0$, define $K_{r}=\{u \in K$ : $\|u\|<r\}$. Assume that $T: \bar{K}_{r} \rightarrow K$ is completely continuous such that $T x \neq x$ for $x \in \partial K_{r}=\{u \in$ $K:\|u\|=r\}$.

(i) If $\|T x\| \geq\|x\|$ for $x \in \partial K_{r}$, then $i\left(T, K_{r}, K\right)=0$.

(ii) If $\|T x\| \leq\|x\|$ for $x \in \partial K_{r}$, then $i\left(T, K_{r}, K\right)=1$.

Now we give our main results on positive periodic solutions for (4.1).

Theorem 4.10. (a) If $\bar{i}_{0}=1$ or 2 , then (4.1) has $\bar{i}_{0}$ positive w-periodic solutions for $\lambda>$ $1 / f_{1}(1) l \int_{0}^{\omega} b(s) d s>0$;

(b) If $\underline{i}_{-\infty}=1$ or 2 , then (4.1) has $\underline{i}_{-\infty}$ positive w-periodic solutions for $0<\lambda<(m-(M+$ $m)|c|) / L M(1-|c|) F(1) \int_{0}^{\omega} b(s) d s$;

(c) If $\bar{i}_{\infty}=0$ or $\underline{i}_{0}=0$, then (4.1) has no positive $\omega$-periodic solutions for sufficiently small or sufficiently large $\lambda>0$, respectively.

Proof. (a) Choose $r_{1}=1$. Take $\lambda_{0}=1 / f_{1}\left(r_{1}\right) l \int_{0}^{\omega} b(s) d s>0$, then for all $\lambda>\lambda_{0}$, we have from Lemma 4.7 that

$$
\|Q y\|>\|y\|, \quad \text { for } y \in \partial K_{r_{1}} .
$$

Case 1. If $\bar{f}_{0}=0$, we can choose $0<\bar{r}_{2}<r_{1}$, so that $f(u) \leq \varepsilon u$ for $0 \leq u \leq \bar{r}_{2}$, where the constant $\varepsilon>0$ satisfies

$$
\lambda \varepsilon \frac{L M \int_{0}^{\omega} b(s) d s}{m-(M+m)|c|}<1
$$

Letting $r_{2}=(1-|c|) \bar{r}_{2}$, we have $f\left(\left(A^{-1} y\right)(t-\tau(t))\right) \leq \varepsilon\left(A^{-1} y\right)(t-\tau(t))$ for $y \in K_{r_{2}}$. By Lemma 2.2, we have $0 \leq\left(A^{-1} y\right)(t-\tau(t)) \leq\|y\| /(1-|c|) \leq \bar{r}_{2}$ for $y \in \partial K_{r_{2}}$. In view of Lemma 4.6 and (4.34), we have for $y \in \partial K_{r_{2}}$ that

$$
\|Q y\| \leq \lambda \varepsilon \frac{L M \int_{0}^{\omega} b(s) d s}{m-(M+m)|c|}\|y\|<\|y\|
$$

It follows from Lemma 4.9 and (4.33) that

$$
i\left(Q, K_{r_{2}}, K\right)=1, \quad i\left(Q, K_{r_{1}}, K\right)=0,
$$


thus $i\left(Q, K_{r_{1}} \backslash \bar{K}_{r_{2}}, K\right)=-1$ and $Q$ has a fixed point $y$ in $K_{r_{1}} \backslash \bar{K}_{r_{2}}$, which means $\left(A^{-1} y\right)(t)$ is a positive $\omega$-positive solution of (4.1) for $\lambda>\lambda_{0}$.

Case 2. If $\bar{f}_{\infty}=0$, there exists a constant $\widetilde{H}>0$ such that $f(u) \leq \varepsilon u$ for $u \geq \widetilde{H}$, where the constant $\varepsilon>0$ satisfies

$$
\lambda \varepsilon \frac{L M \int_{0}^{\omega} b(s) d s}{m-(M+m)|c|}<1
$$

Letting $r_{3}=\max \left\{2 r_{1}, \widetilde{H}\left(1-c^{2}\right) /(\alpha-|c|)\right\}$, we have $f\left(\left(A^{-1} y\right)(t-\tau(t))\right) \leq \varepsilon\left(A^{-1} y\right)(t-\tau(t))$ for $y \in K_{r_{3}}$. By Lemma 2.2, we have $\left(A^{-1} y\right)(t-\tau(t)) \geq\left((\alpha-|c|) /\left(1-c^{2}\right)\right)\|y\| \geq \widetilde{H}$ for $y \in \partial K_{r_{3}}$. Thus by Lemma 4.6 and (4.37), we have for $y \in \partial K_{r_{3}}$ that

$$
\|Q y\| \leq \lambda \varepsilon \frac{L M \int_{0}^{\omega} b(s) d s}{m-(M+m)|c|}\|y\|<\|y\|
$$

Recalling from Lemma 4.9 and (4.33) that

$$
i\left(Q, K_{r_{3}}, K\right)=1, \quad i\left(Q, K_{r_{1}}, K\right)=0,
$$

then $i\left(Q, K_{r_{3}} \backslash \bar{K}_{r_{1}}, K\right)=1$ and $Q$ has a fixed point $y$ in $K_{r_{3}} \backslash \bar{K}_{r_{1}}$, which means $\left(A^{-1} y\right)(t)$ is a positive $\omega$-positive solution of (4.1) for $\lambda>\lambda_{0}$.

Case 3. If $\bar{f}_{0}=\bar{f}_{\infty}=0$, from the above arguments, there exist $0<r_{2}<r_{1}<r_{3}$ such that $Q$ has a fixed point $y_{1}(t)$ in $K_{r_{1}} \backslash \bar{K}_{r_{2}}$ and a fixed point $y_{2}(t)$ in $K_{r_{3}} \backslash \bar{K}_{r_{1}}$. Consequently, $\left(A^{-1} y_{1}\right)(t)$ and $\left(A^{-1} y_{2}\right)(t)$ are two positive $\omega$-periodic solutions of (4.1) for $\lambda>\lambda_{0}$.

(b) Let $r_{1}=1$. Take $\lambda_{0}=(m-(M+m)|c|) / L M(1-|c|) F\left(r_{1}\right) \int_{0}^{\omega} b(s) d s>0$; then by Lemma 4.8 we know if $\lambda<\lambda_{0}$ then

$$
\|Q y\|<\|y\|, \quad y \in \partial K_{r_{1}}
$$

Case 1. If $\underline{f}_{0}=\infty$, we can choose $0<\bar{r}_{2}<r_{1}$ so that $f(u) \geq \eta u$ for $0 \leq u \leq \bar{r}_{2}$, where the constant $\eta>0$ satisfies

$$
\operatorname{ll} \frac{\alpha-|c|}{1-c^{2}} \int_{0}^{\omega} b(s) d s>1
$$

Letting $r_{2}=(1-|c|) \bar{r}_{2}$, we have $f\left(\left(A^{-1} y\right)(t-\tau(t))\right) \geq \eta\left(A^{-1} y\right)(t-\tau(t))$ for $y \in K_{r_{2}}$. By Lemma 2.2, we have $0 \leq\left(A^{-1} y\right)(t-\tau(t)) \leq\|y\| /(1-|c|) \leq \bar{r}_{2}$ for $y \in \partial K_{r_{2}}$. Thus by Lemma 4.5 and (4.41),

$$
\|Q y\| \geq \lambda \ln \frac{\alpha-|c|}{1-c^{2}} \int_{0}^{\omega} b(s) d s\|y\|>\|y\|
$$


It follows from Lemma 4.9 and (4.40) that

$$
i\left(Q, K_{r_{2}}, K\right)=0, \quad i\left(Q, K_{r_{1}}, K\right)=1,
$$

which implies $i\left(Q, K_{r_{1}} \backslash \bar{K}_{r_{2}}, K\right)=1$ and $Q$ has a fixed point $y$ in $K_{r_{1}} \backslash \bar{K}_{r_{2}}$. Therefore, $\left(A^{-1} y\right)(t)$ is a positive $\omega$-periodic solution of (4.1) for $0<\lambda<\lambda_{0}$.

Case 2. If $\underline{f}_{\infty}=\infty$, there exists a constant $\widetilde{H}>0$ such that $f(u) \geq \eta u$ for $u \geq \widetilde{H}$, where the constant $\eta>0$ satisfies

$$
\lambda l \eta \frac{\alpha-|c|}{1-c^{2}} \int_{0}^{\omega} b(s) d s>1
$$

Letting $r_{3}=\max \left\{2 r_{1}, \widetilde{H}\left(1-c^{2}\right) /(\alpha-|c|)\right\}$, we have $f\left(\left(A^{-1} y\right)(t-\tau(t))\right) \geq \eta\left(A^{-1} y\right)(t-\tau(t))$ for $y \in K_{r_{3}}$. By Lemma 2.2, we have $\left(A^{-1} y\right)(t-\tau(t)) \geq\left((\alpha-|c|) /\left(1-c^{2}\right)\right)\|y\| \geq \widetilde{H}$ for $y \in \partial K_{r_{3}}$. Thus by Lemma 4.5 and (4.44), we have for $y \in \partial K_{r_{3}}$ that

$$
\|Q y\| \geq \lambda l \eta \frac{\alpha-|c|}{1-c^{2}} \int_{0}^{\omega} b(s) d s\|y\|>\|y\|
$$

It follows from Lemma 4.9 and (4.40) that

$$
i\left(Q, K_{r_{3}}, K\right)=0, \quad i\left(Q, K_{r_{1}}, K\right)=1 .
$$

that is, $i\left(Q, K_{r_{3}} \backslash \bar{K}_{r_{1}}, K\right)=-1$ and $Q$ has a fixed point $y$ in $K_{r_{3}} \backslash \bar{K}_{r_{1}}$. That means $\left(A^{-1} y\right)(t)$ is a positive $\omega$-periodic solution of (4.1) for $0<\lambda<\lambda_{0}$.

Case 3. If $\underline{f}_{0}=\underline{f}_{-\infty}=\infty$, from the above arguments, $Q$ has a fixed point $y_{1}$ in $K_{r_{1}} \backslash \bar{K}_{r_{2}}$ and a fixed point $y_{2}$ in $K_{r_{3}} \backslash \bar{K}_{r_{1}}$. Consequently, $\left(A^{-1} y_{1}\right)(t)$ and $\left(A^{-1} y_{2}\right)(t)$ are two positive $\omega$ periodic solutions of (4.1) for $0<\lambda<\lambda_{0}$. $t \in[0, \omega]$.

(c) By Lemma 2.2, if $y \in K$, then $\left(A^{-1} y\right)(t-\tau(t)) \geq\left((\alpha-|c|) /\left(1-c^{2}\right)\right)\|y\|>0$ for Case 1. If $\underline{i}_{0}=0$, we have $\underline{f}_{0}>0$ and $\underline{f}_{\infty}>0$. Let $b_{1}=\min \{f(u) / u ; u>0\}>0$, then we obtain

$$
f(u) \geq b_{1} u, \quad u \in[0,+\infty) .
$$

Assume $y(t)$ is a positive $\omega$-periodic solution of (4.1) for $\lambda>\lambda_{0}$, where $\lambda_{0}=\left(1-c^{2}\right) / l b_{1}(\alpha-$ $|c|) \int_{0}^{\omega} b(s) d s>0$. Since $Q y(t)=y(t)$ for $t \in[0, \omega]$, then by Lemma 4.5 , if $\lambda>\lambda_{0}$ we have

$$
\|y\|=\|Q y\| \geq \lambda l b_{1} \frac{\alpha-|c|}{1-c^{2}} \int_{0}^{\omega} b(s) d s\|y\|>\|y\|,
$$

which is a contradiction. 
Case 2. If $\bar{i}_{\infty}=0$, we have $\bar{f}_{0}<\infty$ and $\bar{f}_{\infty}<\infty$. Let $b_{2}=\max \{f(u) / u: u>0\}>0$, then we obtain

$$
f(u) \leq b_{2} u, \quad u \in[0, \infty)
$$

Assume $y(t)$ is a positive $\omega$-periodic solution of (4.1) for $0<\lambda<\lambda_{0}$, where $\lambda_{0}=(m-(M+$ $m)|c|) / b_{2} L M \int_{0}^{\omega} b(s) d s$. Since $Q y(t)=y(t)$ for $t \in[0, \omega]$, it follows from Lemma 4.6 that

$$
\|y\|=\|Q y\| \leq \lambda b_{2} \frac{L M \int_{0}^{\omega} b(s) d s}{m-(M+m)|c|}\|y\|<\|y\|
$$

which is a contradiction.

Theorem 4.11. (a) If there exists a constant $b_{1}>0$ such that $f(u) \geq b_{1} u$ for $u \in[0,+\infty)$, then (4.1) has no positive $\omega$-periodic solution for $\lambda>\left(1-c^{2}\right) / l b_{1}(\alpha-|c|) \int_{0}^{\omega} b(s) d s$.

(b) If there exists a constant $b_{2}>0$ such that $f(u) \leq b_{2} u$ for $u \in[0,+\infty)$, then (4.1) has no positive $\omega$-periodic solution for $0<\lambda<(m-(M+m)|c|) / b_{2} L M \int_{0}^{\omega} b(s) d s$.

Proof. From the proof of (c) in Theorem 4.10, we obtain this theorem immediately.

Theorem 4.12. Assume $\underline{i}_{0}=\bar{i}_{0}=\underline{i}_{\infty}=\bar{i}_{\infty}=0$ and that one of the following conditions holds:
(1) $\bar{f}_{0} \leq \underline{f}_{\infty} ;$
(2) $\underline{f}_{0}>\bar{f}_{\infty}$;
(3) $\underline{f}_{0} \leq \underline{f}_{\infty} \leq \bar{f}_{0} \leq \bar{f}_{\infty}$;
(4) $\underline{f}_{-\infty} \leq \underline{f}_{0} \leq \bar{f}_{\infty} \leq \bar{f}_{0}$.

If

$$
\frac{1-c^{2}}{l(\alpha-|c|) \int_{0}^{\omega} b(s) d s \max \left\{\underline{f}_{0^{\prime}}, \bar{f}_{0^{\prime}}, \underline{f}_{\infty}, \bar{f}_{\infty}\right\}}<\lambda<\frac{m-(M+m)|c|}{\operatorname{LM} \int_{0}^{\omega} b(s) d s \min \left\{\underline{f}_{0^{\prime}} \bar{f}_{0^{\prime}}, \underline{f}_{-\infty}, \bar{f}_{\infty}\right\}}
$$

then (4.1) has one positive w-periodic solution.

Proof. We have the following cases.

Case 1. If $\bar{f}_{0} \leq \underline{f}_{\infty}$, then

$$
\frac{1-c^{2}}{\bar{f}_{\infty} l(\alpha-|c|) \int_{0}^{\omega} b(s) d s}<\lambda<\frac{m-(M+m)|c|}{\underline{f}_{0} L M \int_{0}^{\omega} b(s) d s} .
$$


It is easy to see that there exists an $0<\varepsilon<f_{\infty}$ such that

$$
\frac{1-c^{2}}{\left(\bar{f}_{\infty}-\varepsilon\right) l(\alpha-|c|) \int_{0}^{\omega} b(s) d s}<\lambda<\frac{m-(M+m)|c|}{\left(\underline{f}_{0}+\varepsilon\right) L M \int_{0}^{\omega} b(s) d s} .
$$

For the above $\varepsilon$, we choose $\bar{r}_{1}>0$ such that $f(u) \leq\left(\underline{f}_{0}+\varepsilon\right) u$ for $0 \leq u \leq \bar{r}_{1}$. Letting $r_{1}=$ $(1-|c|) \bar{r}_{1}$, we have $f\left(\left(A^{-1} y\right)(t-\tau(t))\right) \leq\left(\underline{f}_{0}+\varepsilon\right)\left(A^{-1} y\right)(t-\tau(t))$ for $y \in K_{r_{1}}$. By Lemma 2.2, we have $0 \leq\left(A^{-1} y\right)(t-\tau(t)) \leq\|y\| /(1-|c|) \leq \bar{r}_{1}$ for $K \in \partial K_{r_{1}}$. Thus by Lemma 4.6 we have for $y \in \partial K_{r_{1}}$ that

$$
\|Q y\| \leq \lambda\left(\underline{f}_{0}+\varepsilon\right) \frac{L M \int_{0}^{\omega} b(s) d s}{m-(M+m)|c|}\|y\|<\|y\|
$$

On the other hand, there exists a constant $\widetilde{H}>0$ such that $f(u) \geq\left(\bar{f}_{\infty}-\varepsilon\right) u$ for $u \geq \widetilde{H}$. Letting $r_{2}=\max \left\{2 r_{1}, \widetilde{H}\left(1-c^{2}\right) /(\alpha-|c|)\right\}$, we have $f\left(\left(A^{-1} y\right)(t-\tau(t))\right) \geq\left(\bar{f}_{\infty}-\varepsilon\right)\left(A^{-1} y\right)(t-\tau(t))$ for $y \in K_{r_{2}}$. By Lemma 2.2, we have $\left(A^{-1} y\right)(t-\tau(t)) \geq\left((\alpha-|c|) /\left(1-c^{2}\right)\right)\|y\| \geq \widetilde{H}$ for $y \in \partial K_{r_{2}}$. Thus by Lemma 4.5 , for $y \in \partial K_{r_{2}}$

$$
\|Q y\| \geq \lambda l\left(\bar{f}_{\infty}-\varepsilon\right) \frac{\alpha-|c|}{1-c^{2}} \int_{0}^{\omega} b(s) d s\|y\|>\|y\|
$$

It follows from Lemma 4.9 that

$$
i\left(Q, K_{r_{1}}, K\right)=1, \quad i\left(Q, K_{r_{2}}, K\right)=0,
$$

thus $i\left(Q, K_{r_{2}} \backslash \bar{K}_{r_{1}}, K\right)=-1$ and $Q$ has a fixed point $y$ in $K_{r_{2}} \backslash \bar{K}_{r_{1}}$. So $\left(A^{-1} y\right)(t)$ is a positive $\omega$-periodic solution of (4.1).

Case 2. If $\underline{f}_{0}>\bar{f}_{\infty}$, in this case, we have

$$
\frac{1-c^{2}}{\bar{f}_{0} l(\alpha-|c|) \int_{0}^{\omega} b(s) d s}<\lambda<\frac{m-(M+m)|c|}{\underline{f}_{-\infty} L M \int_{0}^{\omega} b(s) d s}
$$

It is easy to see that there exists an $0<\varepsilon<f_{0}$ such that

$$
\frac{1-c^{2}}{\left(\bar{f}_{0}-\varepsilon\right) l(\alpha-|c|) \int_{0}^{\omega} b(s) d s}<\lambda<\frac{m-(M+m)|c|}{\left(\underline{f}_{\infty}+\varepsilon\right) L M \int_{0}^{\omega} b(s) d s} .
$$

For the above $\varepsilon$, we choose $\bar{r}_{1}>0$ such that $f(u) \geq\left(\bar{f}_{0}-\varepsilon\right) u$ for $0 \leq u \leq \bar{r}_{1}$. Letting $r_{1}=$ $(1-|c|) \bar{r}_{1}$, we have $f\left(\left(A^{-1} y\right)(t-\tau(t))\right) \geq\left(\bar{f}_{0}-\varepsilon\right)\left(A^{-1} y\right)(t-\tau(t))$ for $y \in K_{r_{1}}$. By Lemma 2.2, 
we have $0 \leq\left(A^{-1} y\right)(t-\tau(t)) \leq\|y\| /(1-|c|) \leq \bar{r}_{1}$ for $y \in \partial K_{r_{1}}$. Thus we have by Lemma 4.5 that for $y \in \partial K_{r_{1}}$

$$
\|Q y\| \geq \lambda l\left(\bar{f}_{0}-\varepsilon\right) \frac{\alpha-|c|}{1-c^{2}} \int_{0}^{\omega} b(s) d s\|y\|>\|y\| .
$$

On the other hand, there exists a constant $\widetilde{H}>0$ such that $f(u) \leq\left(\underline{f}_{\infty}+\varepsilon\right) u$ for $u \geq \widetilde{H}$. Letting $r_{2}=\max \left\{2 r_{1}, \widetilde{H}\left(1-c^{2}\right) /(\alpha-|c|)\right\}$, we have $f\left(\left(A^{-1} y\right)(t-\tau(t))\right) \leq\left(\underline{f}_{-\infty}+\varepsilon\right)\left(A^{-1} y\right)(t-\tau(t))$ for $y \in K_{r_{2}}$. By Lemma 2.2 we have $\left(A^{-1} y\right)(t-\tau(t)) \geq\left((\alpha-|c|) /\left(1-c^{2}\right)\right)\|y\| \geq \widetilde{H}$ for $y \in \partial K_{r_{2}}$. Thus by Lemma 4.6 , for $y \in \partial K_{r_{2}}$

$$
\|Q y\| \leq \lambda\left(\underline{f}_{\infty}+\varepsilon\right) \frac{L M \int_{0}^{\omega} b(s) d s}{m-(M+m)|c|}\|y\|
$$

It follows from Lemma 4.9 that

$$
i\left(Q, K_{r_{1}}, K\right)=0, \quad i\left(Q, K_{r_{2}}, K\right)=1
$$

Thus $i\left(Q, K_{r_{2}} \backslash \bar{K}_{r_{1}}, K\right)=-1$ and $Q$ has a fixed point $y$ in $K_{r_{2}} \backslash \bar{K}_{r_{1}}$, proving that $\left(A^{-1} y\right)(t)$ is a positive $\omega$-periodic solution of (4.1).

Case 3. One has $\underline{f}_{0} \leq \underline{f}_{\infty} \leq \bar{f}_{0} \leq \bar{f}_{\infty}$. The proof is the same as in Case 1 .

Case 4. One has $\underline{f}_{\infty} \leq \underline{f}_{0} \leq \bar{f}_{\infty} \leq \bar{f}_{0}$. The proof is the same as in Case 2 .

\subsection{Case II}

Assume $c>0$ and $c<\min \{m /(M+m),(L M-l m) /(L-l) M-l m\}$.

Define

$$
f_{2}(r)=\min \left\{f(t): \frac{\alpha}{1-c} r \leq t \leq \frac{r}{1-c}\right\}
$$

Similarly as in Section 4.1, we get the following results.

Theorem 4.13. (a) If $\bar{i}_{0}=1$ or 2 , then (4.1) has $i_{0}$ positive w-periodic solutions for $\lambda>$ $1 / f_{2}(1) l \int_{0}^{\omega} b(s) d s>0$.

(b) If $\underline{i}_{\infty}=1$ or 2 , then (4.1) has $i_{\infty}$ positive w-periodic solutions for $0<\lambda<(m-(M+$ $m) c) / L M(1-c) F(1) \int_{0}^{\omega} b(s) d s$.

(c) If $\bar{i}_{\infty}=0$ or $\underline{i}_{0}=0$, then (4.1) has no positive $\omega$-periodic solution for sufficiently small or large $\lambda>0$, respectively.

Theorem 4.14. (a) If there exists a constant $b_{1}>0$ such that $f(u) \geq b_{1} u$ for $u \in[0,+\infty)$, then (4.1) has no positive $\omega$-periodic solution for $\lambda>(1-c) / l \alpha b_{1} \int_{0}^{\omega} b(s) d s$. 
(b) If there exists a constant $b_{2}>0$ such that $f(u) \leq b_{2} u$ for $u \in[0,+\infty)$, then (4.1) has no positive $\omega$-periodic solution for $0<\lambda<(m-(M+m) c) / b_{2} L M \int_{0}^{\omega} b(s) d s$.

Theorem 4.15. Assume $\underline{i}_{0}=\bar{i}_{0}=\underline{i}_{\infty}=\bar{i}_{\infty}=0$ hold and that one of the following conditions holds:
(1) $\bar{f}_{0} \leq \underline{f}_{\infty} ;$
(2) $\underline{f}_{0}>\bar{f}_{\infty}$;
(3) $\underline{f}_{0} \leq \underline{f}_{-} \leq \bar{f}_{0} \leq \bar{f}_{\infty}$;
(4) $\underline{f}_{-\infty} \leq \underline{f}_{0} \leq \bar{f}_{\infty} \leq \bar{f}_{0}$.

If

$$
\frac{1-c}{l \alpha \int_{0}^{\omega} b(s) d s \max \left\{\underline{f}_{0^{\prime}}, \bar{f}_{0^{\prime}}, \underline{f}_{-}, \bar{f}_{\infty}\right\}}<\lambda<\frac{m-(M+m) c}{L M \int_{0}^{\omega} b(s) d s \min \left\{\underline{f}_{0^{\prime}}, \bar{f}_{0^{\prime}}, \underline{f}_{-}, \bar{f}_{\infty}\right\}}
$$

then (4.1) has one positive w-periodic solution.

Remark 4.16. In a similar way, one can consider the second-order neutral functional differential equation $(x(t)-c x(t-\delta(t)))^{\prime \prime}-a(t) x(t)=-\lambda b(t) f(x(t-\tau(t)))$.

\section{Examples}

Example 5.1. Consider the following equation:

$$
\left(x(t)-15 x\left(t-\frac{1}{60} \sin 4 t\right)\right)^{\prime \prime}=x^{\prime}(t) \sin 4 t+\arctan \left(\frac{x(t-\sin 4 t)}{1+\cos ^{3}(4 t)}\right)+\cos 4 t .
$$

Comparing (5.1) to (3.1), we have $\omega=\pi / 2, f(t, x)=x(t) \sin 4 t, g(t, x)=\arctan (x /(1+$ $\left.\left.\cos ^{3}(4 t)\right)\right), c=15, \delta(t)=(1 / 60) \sin 4 t, \tau(t)=\sin 4 t, e(t)=\cos 4 t$ and $\delta_{1}=\max _{t \in[0, \omega]} \mid(1 /$ 15) $\cos 4 t \mid=1 / 15$, and we can easily choose $D>\pi / 2$ and $M=\pi / 2$ such that $\left(H_{2}\right)$ and $\left(H_{3}\right)$ holds. Regarding assumption $\left(H_{1}\right)$ note that

$$
\left|f\left(t, x^{\prime}(t)\right)\right| \leq\left|x^{\prime}(t)\right|
$$

that is, $\left(H_{1}\right)$ holds with $K_{1}=1, b=0$, and

$$
\frac{\omega^{1 / 2}(1+|c|)^{1 / 2} \sqrt{2 K_{1}}}{|1-| c||-|c| \delta_{1}}=\frac{\sqrt{\pi / 2}(1+15)^{1 / 2} \sqrt{2}}{|1-15|-(1 / 15) \cdot 15}=\frac{4 \sqrt{\pi}}{13}<1
$$

Hence by Theorem 3.2, (5.1) has at least one $\pi / 2$-periodic solution. 
Example 5.2. Consider the following neutral functional differential equation:

$$
\left(u(t)+\frac{7}{30} u(t-\sin t)\right)^{\prime \prime}+\frac{1}{16} u(t)=\lambda(1-\sin t) u^{2}(t-\tau(t)) a^{u(t-\tau(t))},
$$

where $\lambda$ and $0<a<1$ are two positive parameters, $\tau(t+2 \pi)=\tau(t)$.

Comparing (5.4) to (4.1), we see that $\delta(t)=\sin t, c=-7 / 30, a(t) \equiv 1 / 16, b(t)=$ $1-\sin t, \omega=2 \pi, f(u)=u^{2} a^{u}$. Clearly, $M=1 / 16<(\pi / 2 \pi)^{2}=1 / 4, \bar{f}_{0}=0, \bar{f}_{\infty}=0, \bar{i}_{0}=2$. By Theorem 4.10, we easily get the following conclusion: (5.4) has two positive $\omega$-periodic solutions for $\lambda>1 / 4 \pi r_{1}$, where $r_{1}=\min \{f(0.27), f(30 / 23)\}$.

In fact, by simple computations, we have

$$
\begin{gathered}
M=m=\frac{1}{16}, \quad \beta=\frac{1}{4}, \quad L=\frac{1}{2 \beta \sin (\beta 2 \pi / 2)}=2 \sqrt{2}, \quad l=\frac{\cos (\beta 2 \pi / 2)}{(2 \beta \sin (\beta 2 \pi / 2))}=2, \\
k=\frac{2+\sqrt{2}}{8}, \quad k_{1}=\frac{\sqrt{2}+1-\sqrt{3}}{2}, \quad \alpha=\frac{8}{23} \sqrt{2}, \\
|c|=\frac{7}{30}<\min \left\{k_{1}, \frac{m}{M+m}\right\}=\frac{\sqrt{2}+1-\sqrt{3}}{2}, \quad|c|=\frac{7}{30}<\frac{8}{23} \sqrt{2}=\alpha, \\
f_{1}(1)=\min \left\{f(t): 0.27 \approx \frac{(8 / 23) \sqrt{2}-(7 / 30)}{1-(7 / 30)^{2}} \leq t \leq \frac{30}{23}\right\}=\min \left\{f(0.27), f\left(\frac{30}{23}\right)\right\}=r_{1}, \\
\frac{1}{f_{1}(1) l \int_{0}^{\omega} b(s) d s}=\frac{1}{4 \pi r_{1}} .
\end{gathered}
$$

\section{Acknowledgment}

This research is supported by the National Natural Science Foundation of China (no. 10971202).

\section{References}

[1] M. R. Zhang, "Periodic solutions of linear and quasilinear neutral functional differential equations," Journal of Mathematical Analysis and Applications, vol. 189, no. 2, pp. 378-392, 1995.

[2] S. Lu and W. Ge, "Periodic solutions of neutral differential equation with multiple deviating arguments," Applied Mathematics and Computation, vol. 156, no. 3, pp. 705-717, 2004.

[3] Q. Wang and B. Dai, "Three periodic solutions of nonlinear neutral functional differential equations," Nonlinear Analysis. Real World Applications, vol. 9, no. 3, pp. 977-984, 2008.

[4] J. Wu and Z. Wang, "Two periodic solutions of second-order neutral functional differential equations," Journal of Mathematical Analysis and Applications, vol. 329, no. 1, pp. 677-689, 2007.

[5] J. Wu and Y. Liu, "Two periodic solutions of neutral difference systems depending on two parameters," Journal of Computational and Applied Mathematics, vol. 206, no. 2, pp. 713-725, 2007.

[6] S. Lu and W. Ge, "Existence of periodic solutions for a kind of second-order neutral functional differential equation," Applied Mathematics and Computation, vol. 157, no. 2, pp. 433-448, 2004.

[7] W. S. Cheung, J. Ren, and W. Han, "Positive periodic solution of second-order neutral functional differential equations," Nonlinear Analysis. Theory, Methods \& Applications, vol. 71, no. 9, pp. 3948-3955, 2009. 
[8] R. E. Gaines and J. L. Mawhin, Coincidence Degree, and Nonlinear Differential Equations, Springer, Berlin, Germany, 1977.

[9] M. Krasnoselskii, Positive Solutions of Operator Equations, P. Noordhoff Ltd., Groningen, The Netherlands, 1964. 


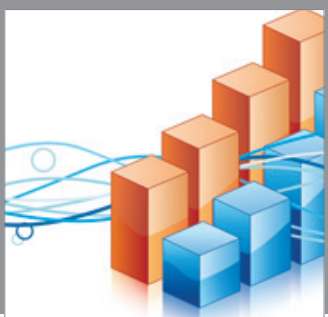

Advances in

Operations Research

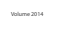

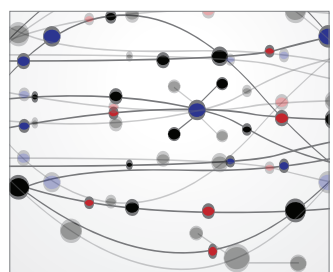

\section{The Scientific} World Journal
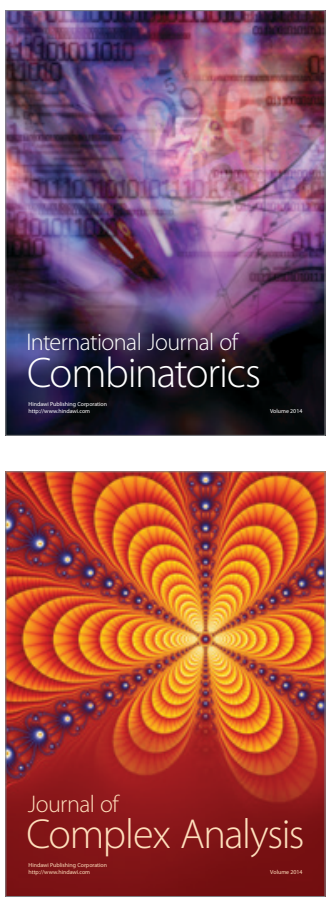

International Journal of

Mathematics and

Mathematical

Sciences
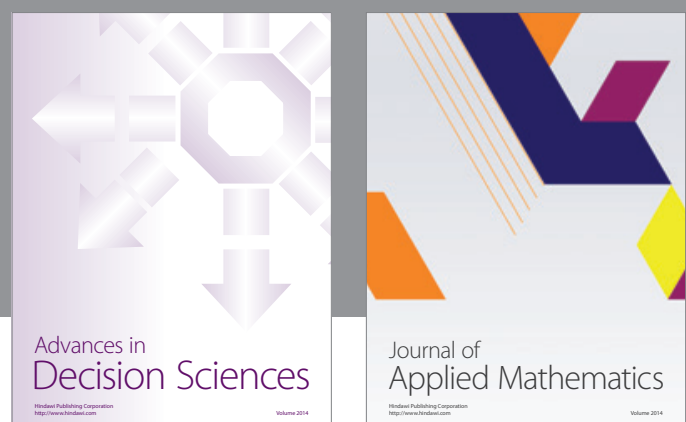

Journal of

Applied Mathematics
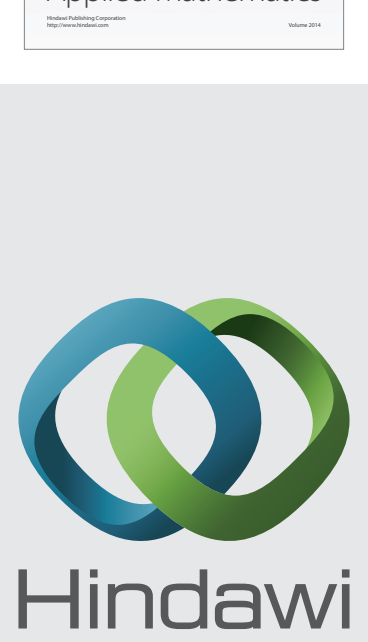

Submit your manuscripts at http://www.hindawi.com
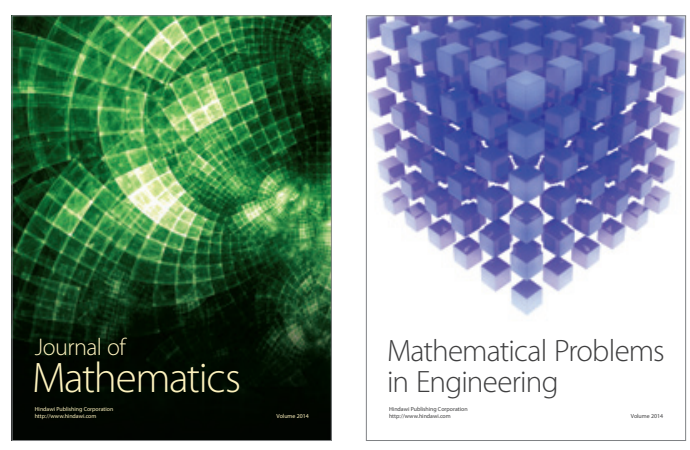

Mathematical Problems in Engineering
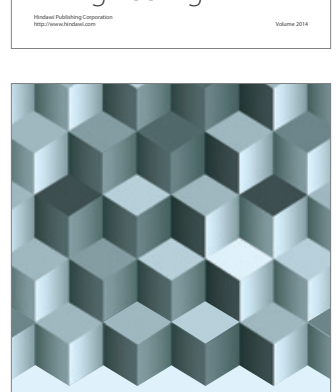

Journal of

Function Spaces
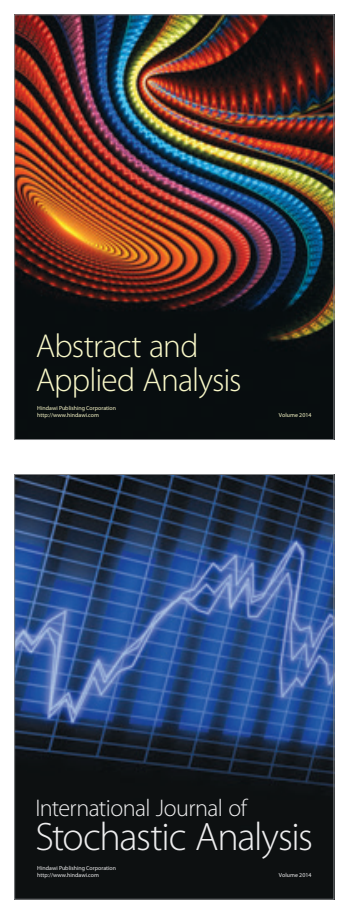

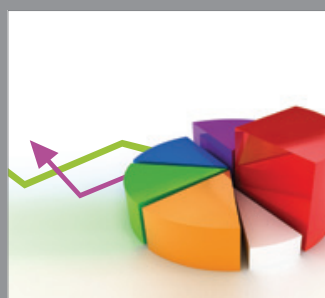

ournal of

Probability and Statistics

Promensencen
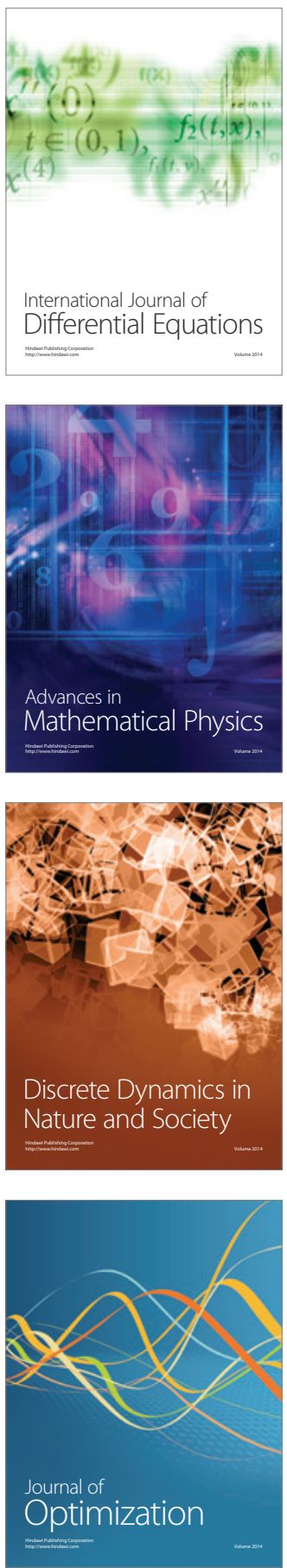